\title{
Adaptación del Test Stroop Victoria en Estudiantes Ecuatorianos
}

\author{
Adaptation of Victoria Stroop Test in Ecuadorians Students
}

\author{
Carlos Ramos ${ }^{1}$
}

\section{Resumen}

La evaluación neuropsicológica del control inhibitorio puede ser realizada mediante tareas de tipo específicas (reactivos creados para evaluar una determinada función neuropsicológica), no específicas (reactivos creados con algún fin diferente y que pueden servir para evaluar una función neuropsicológica) y de exploración diferida (escalas de observación conductual). En el contexto del primer tipo de tareas se ha desarrollado el experimento Stroop Victoria. Objetivo. En el presente estudio se tuvo como finalidad: (a) adaptar el contenido lingüístico del test Stroop Victoria mediante el uso del Sistema de Construcción de Experimentos en Psicología - PEBL, (b) relacionar las medidas obtenidas en el test con las de una escala que valore un constructo similar, y (c) comparar y relacionar las medidas obtenidas en el experimento según el género y el grupo etario de los participantes. Método. Estudio de tipo cuantitativo transeccional. Los instrumentos de medida fueron el experimento Stroop Victoria y la escala BIS 11-c. Resultados. Se encontró correlación entre las medidas del experimento Stroop Victoria (errores de impulsividad y control inhibitorio). Los errores de impulsividad del experimento están asociados a las dificultades de impulsividad motora de la BIS 11-c. No se encontró relación entre el género, edad de los participantes y los resultados obtenidos en el experimento. Conclusiones. Las medidas de impulsividad pertenecerían a un sistema denominado dirimidor de conflictos, con el que el ser humano actúa de forma automática o semiconsciente, mientras que los errores en el control inhibitorio estarían asociados a déficit en el sistema atencional supervisor.

Palabras clave: adaptación del Test Stroop, control inhibitorio, impulsividad, psicología experimental, Stroop

\begin{abstract}
Neuropsychological assessment of inhibitory control may be accomplished by specific tasks (reagents created to assess neuropsychological function of interest) not specific tasks (reagents created with a different purpose and can be used to assess a particular neuropsychological function) and deferred exploration (scales of behavior observation). In the first type of tasks it has been developed the Victoria Stroop experiment. Aim. To adapt the Victoria Stroop test in a sample of Ecuadorian students. Method. The design of research was a quantitative transeccional study. The measuring instruments were the experiment Stroop Victoria and BIS 11-c scale. Furthermore, the relationship between the scores of the experiment and the sociodemographic variables were analyzed. Results. A significant correlation between measurements of Stroop Victoria (impulsivity and inhibitory control) was found. Impulsiveness errors of the experiment are associated with the motor impulsivity BIS 11-c. It were not found relationship between gender, age of participants and the results obtained in the experiment. Conclusions. Impulsivity measures belong to a system called dirimidor conflict, where human beings act automatically or semi-conscious, whereas intrusion errors inhibitory control failure would be associated with deficits in attentional system supervisor.
\end{abstract}

Keywords: experimental psychology, impulsivity, inhibitory control, Stroop

\footnotetext{
${ }^{1} \mathrm{PhD}$ en Psicología. Neuropsicólogo Clínico. Profesor Investigador de la Universidad Indoamérica, Quito-Ecuador. Profesor Investigador de la Escuela de Psicología de la Universidad Internacional SEK Ecuador, Quito-Ecuador. Correo: ps_carlosramos@hotmail.com
} 


\section{Introducción}

Las funciones ejecutivas engloban diversas habilidades mentales que permiten a un individuo actuar de forma regulada, llevar a cabo un comportamiento eficaz y creativo, tomando en cuenta objetivos personales y en parámetros socialmente aceptados (Lezak, 1995).

Goldberg (2002) propone una interesante analogía en relación con el papel de las funciones ejecutivas en el trabajo cerebral de la cognición y comportamiento. Según el autor, las funciones ejecutivas cumplirían el papel de ser un director de orquesta, donde que supervisa, coordina y dirige todas las habilidades mentales que ejecutan la actuación neurocognitiva.

No hay un consenso respecto de la forma en que se organizan las funciones ejecutivas. Para algunos autores, la organización respondería a factores únicos o múltiples. En el primer caso, por ejemplo, se ha descrito que las funciones ejecutivas se agruparían en un factor único como el sistema atencional supervisor (Norman \& Shallice, 1986) o en el bucle ejecutivo central (Baddeley, 2012). Otros autores como Gioia, Isquith, Retzlaff y Espy (2002) afirman que las funciones ejecutivas se agruparían en varios factores como la regulación emocional, regulación comportamental y metacognición.

Brown (2008) y Anderson y Reidy (2012) han propuesto la existencia diversas funciones ejecutivas, las cuales guardan relación con el funcionamiento del lóbulo frontal y muestran un importante aporte en cuanto a la regulación consciente de la cognición y el comportamiento humano (Damasio, 1994). Dichas funciones ejecutivas son: memoria de trabajo, flexibilidad cognitiva, control inhibitorio, planificación, iniciativa, organización de materiales, regulación de la motivación y el estado de alerta, control emocional, monitorización, establecimiento de metas y el control atencional.

Según Barkley (2011) la función ejecutiva con mayor relevancia en el proceso de autorregulación del comportamiento es el control inhibitorio, ya que cumple con el rol central de inhibir la tendencia de actuar de forma automática, para luego dar paso al procesamiento cognitivo complejo que supone el mantenimiento de la información online mientras se resuelve algún problema, se supervisa conscientemente la actividad, se regula el estado de alerta, las emociones y la motivación y/o se auto-dirige el comportamiento mediante el lenguaje interno.

Según Diamond (2006 mencionado en Rubiales, 2014), el control inhibitorio permite al ser humano inhibir una respuesta comportamental impulsiva ante un determinado estímulo, suprimir la información irrelevante mientras se realiza una determinada actividad, lo cual le permite a un individuo desempeñarse de forma prolongada en una actividad sin presentar distracciones.

En el proceso de evaluación del control inhibitorio existen tres estrategias metodológicas basadas en (a) pruebas de tipo específicas, (b) pruebas no específicas y (c) pruebas de exploración diferida. El primer tipo de pruebas consiste en tareas experimentales que han sido desarrolladas para la evaluación exclusiva del control inhibitorio, tales como: los experimentos Go/No-Go o Simon, el test de Stroop, el paradigma Stop, entre otras (García-Gómez, 2015; Ramos, Guevara, Bolaños, \& Ramos, 2015; Verbruggen, Logan, \& Stevens, 2008).

En el segundo tipo de estrategias se encuentran las pruebas de tipo no específico, que consisten en tareas que no han sido desarrolladas para la evaluación del control inhibitorio como tal, no obstante, son interesantes reactivos para su evaluación. El mejor ejemplo del uso de esta segunda estrategia es la aplicación de las subescalas del test de inteligencia de Wechsler como medidas para la evaluación del control inhibitorio o en la evaluación neuropsicológica en general, como por ejemplo, en la medición de la monitorización, planificación, control atencional, entre otras (García-Gómez, 2015; Ramírez \& Rosas, 2007).

Las pruebas de exploración diferida se basan en la observación y reporte del dominio conductual. Consisten en cuestionarios y/o escalas de observación del sujeto. El objetivo es tener un parámetro ecológico del funcionamiento neuropsicológico del control inhibitorio en la vida diaria y en los diversos contextos en los cuales se desenvuelve la persona. Son varios los instrumentos que utilizan esta estrategia de evaluación, como por ejemplo, los cuestionarios para evaluar las funciones ejecutivas: BRIEF 
(Behavior Rating Inventory of Executive Function) desarrollado por Gioia, Isquith, Guy y Kenworthy (2000) o el EFECO propuesto por García-Gómez (2015).

Entre los instrumentos de evaluación del control inhibitorio mediante reactivos específicos, el Sistema de Construcción de Experimentos en Psicología - PEBL (Mueller \& Piper, 2014) permite evaluar dicho aspecto mediante tareas experimentales. Una de las más importantes es el experimento Stroop en su versión Victoria. El principal beneficio del PEBL es su código abierto y su uso libre, que permite al investigador adaptar el experimento a las necesidades de sus hipótesis y objetivos. Por otro lado, la principal limitación del sistema es que ha sido desarrollado para su uso en contextos de habla inglesa y no existen estudios previos de su adaptación en países de habla hispana.

Dentro del contexto descrito, surge como objetivo general el adaptar en test Stroop al contexto ecuatoriano, como objetivos específicos se plantean: (a) adaptar el contenido lingüístico del test Stroop Victoria mediante el uso del Sistema de Construcción de Experimentos en Psicología - PEBL (Mueller \& Piper, 2014), (b) relacionar las medidas obtenidas en el test con las de una escala que valore un constructo similar, y (c) comparar y relacionar las medidas obtenidas en el experimento según el género y el grupo etario de los participantes.

\section{Método}

\section{Participantes}

La muestra estuvo conformada por 81 estudiantes entre 6 y 15 años de edad, pertenecientes al sistema público educativo de Ecuador, 47 hombres (58\%) y 34 mujeres (42\%), la media de edad de los hombres fue 9.85 años (DE 2,73), en las mujeres la media fue 10,41 años (DE 2,24). El nivel educativo de los y las participantes va desde tercero de educación básica hasta primero de bachillerato.

\section{Diseño de la investigación}

El enfoque es cuantitativo, el diseño y alcance del estudio es descriptivo y correlacional, con una temporalidad de tipo transeccional (Hernández, Fernández, \& Baptista, 2014).

\section{Instrumentos y técnicas de medición}

Experimento Stroop Victoria. La tarea experimental Stroop Victoria (Mueller \& Piper, 2014) es una tarea de control inhibitorio basada en el paradigma Stroop clásico (Troyer, Leach, \& Strauss, 2006), la cual está desarrollada en una versión informatizada, en donde se utiliza un computador para poder realizarlo. Esta tarea consta de 3 fases (cada una de 26 estímulos) (Charchat-Fichman \& Martins, 2009). En la primera fase el participante debe presionar los botones adecuados que indiquen el color (azul, rojo, amarillo y verde) de los círculos presentados en el monitor (ver Figura 1).

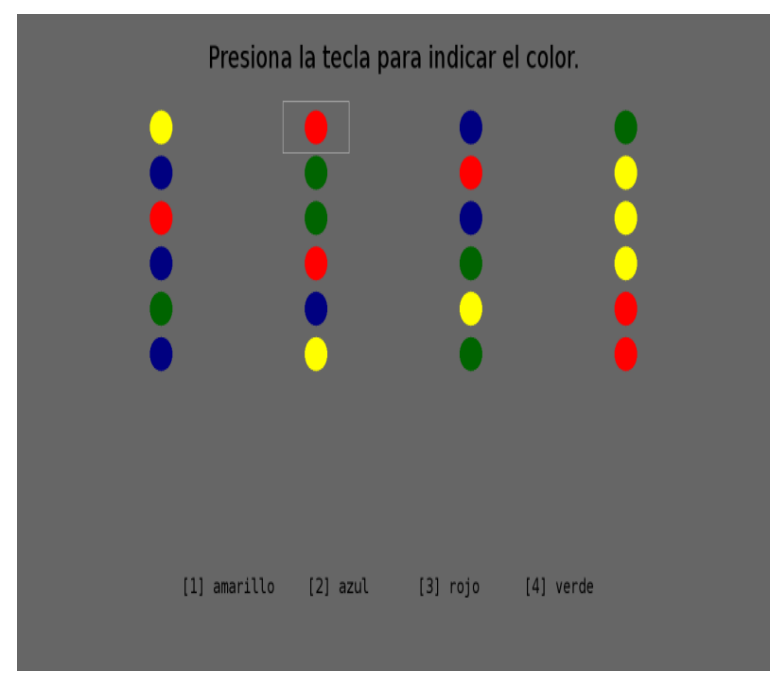

Figura 1. Captura de pantalla de la primera fase del experimento Stroop Victoria. El participante debe seleccionar la opción que indique el color de cada círculo

En la segunda fase del experimento se debe presionar el botón correspondiente al color de la tinta con la cual se encuentra escrita la palabra, que es una diferente al nombre del color y no genera algún efecto de interferencia (ver Figura 2).

En la tercera fase se presenta la interferencia propiamente dicha, ya que se presentan las palabras con un contenido diferente al color de la tinta con el que está escrita (ver figura 3). En esta fase el participante debe inhibir la tendencia de respuesta automática. Por ejemplo, una palabra puede estar pintada de color amarillo y decir azul, 


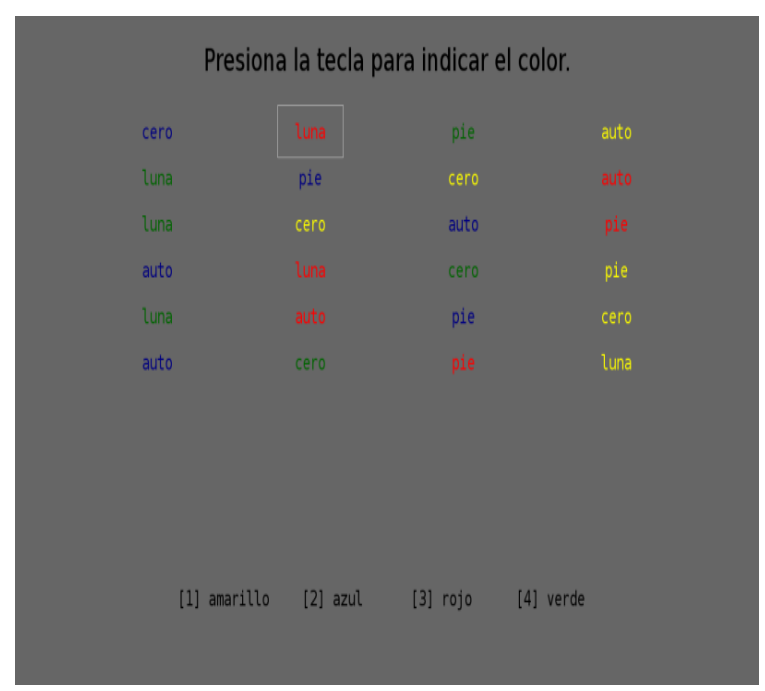

Figura 2. Captura de pantalla de la segunda fase del experimento Stroop Victoria. En ésta el participante debe seleccionar el color de la palabra

siendo el control inhibitorio la función que permitiría al participante inhibir la tendencia automática de leer la palabra y responder amarillo, cuando la respuesta es azul (Malek, Hekmati, Amiri, Pirzadeh, \& Gholizadeh, 2013).

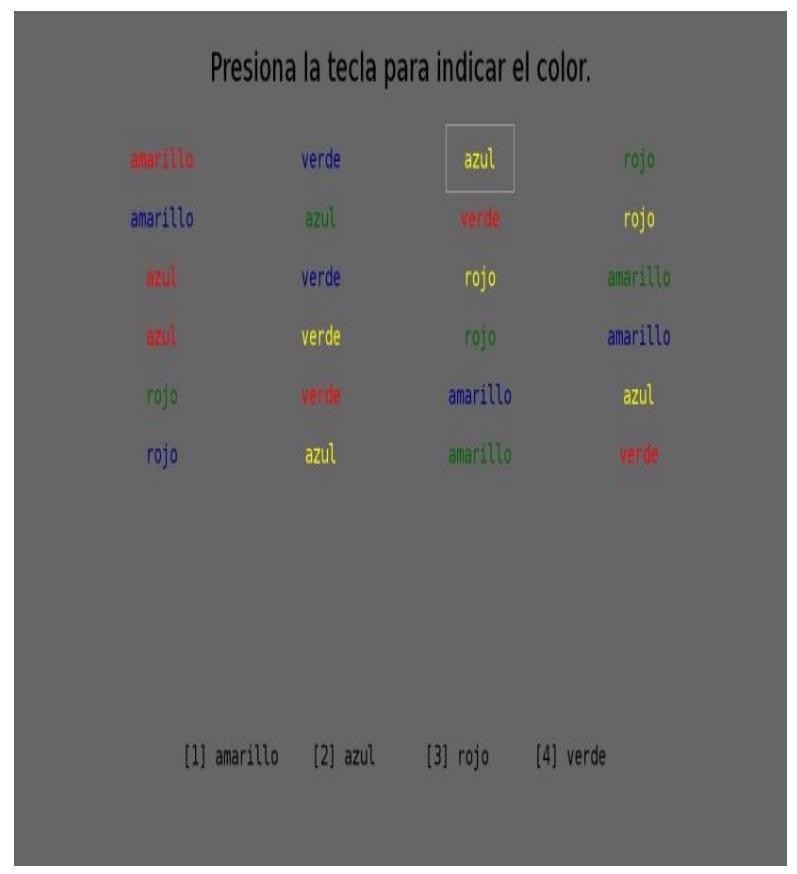

Figura 3. Captura de pantalla de la tercera fase del experimento. En la figura se observa una palabra encerrada en un cuadrado, ésta dice azul y es de color amarillo, en este caso el participante debe seleccionar la opción amarillo que es el color de la palabra e inhibir la tendencia a leer y no seleccionar la respuesta azul. Los fallos en estas respuestas se consideraron como errores de intrusión.
En este experimento se midieron dos variables: (a) errores de impulsividad y (b) errores de intrusión por fallos en el control inhibitorio. El primer tipo de errores consistió en las respuestas fallidas, no existió, en este caso, una conciencia en cuanto a la certeza de la respuesta ( $\sin$ presencia de la intrusión), es decir, por ejemplo, en las tres fases, ante un estímulo de un determinado color se presiona erróneamente otro color.

El segundo tipo de errores se caracterizó por fallos del control inhibitorio, que se pueden observar exclusivamente en la tercera fase de la tarea, ya que a diferencia de las dos fases previas, el participante cuando presiona la opción que indica el contenido textual de la palabra, en vez del color, se produce porque no inhibió la tendencia automática de leer. A cada respuesta de este tipo se la consideró error de intrusión.

\section{Escala BIS 11-c}

La escala de Impulsividad de Barrat BIS 11-c es un cuestionario de auto-reporte que permite evaluar las variables: (a) impulsividad cognitiva, (b) impulsividad asociada a la planificación e (c) impulsividad motora (Stanford et al., 2009). Este instrumento consta de 26 ítems que son evaluados con cero puntos cuando la conducta tiene una frecuencia de "nunca", con un punto cuando es "a veces", con dos puntos cuando es "regularmente" y con tres puntos cuando la conducta se presenta "siempre". El parámetro de consistencia interna del instrumento para impulsividad cognitiva es $\alpha=.59$, impulsividad motora $\alpha=.74$ e impulsividad asociada a la planificación $\alpha=.72$ (Chahin, Cosi, Lorenzo-Seva, \& Vigil-Colet, 2010).

\section{Análisis de datos}

Se analizaron los datos utilizando estadística descriptiva, medidas de tendencia central y dispersión, para describir los valores obtenidos por los participantes. Posteriormente se aplicó correlación de Spearman para analizar la relación entre las variables (a) total de errores por impulsividad, (b) errores de intrusión por fallos en el control inhibitorio, (c) impulsividad motora, (d) impulsividad asociada a la planificación, y (e) impulsividad cognitiva. Con la prueba de Chi cuadrado se analizó si existe alguna asociación entre la edad y las medidas del experimento. 
Además, se aplicó la prueba de U de MannWhitney con la finalidad de analizar si existen diferencias entre géneros.

\section{Procedimiento}

En primer lugar, se tradujeron las instrucciones y los estímulos del experimento, ya que la versiòn original está en inglés. Este proceso fue realizado mediante la manipulación del código de fuente del sistema PEBL (Mueller, 2015). Una vez cumplido con este primer paso, se solicitó el juicio experto de tres profesionales con grado de maestría en neuropsicología, experiencia en la evaluación de las funciones ejecutivas y en la adaptación de reactivos psicométricos al contexto ecuatoriano. Una vez realizadas las modificaciones sugeridas por los expertos, se procedió a realizar un estudio piloto con cinco estudiantes, que no formaron parte del estudio y que pertenecían al mismo grupo etario y tenían las mismas características de los participantes del grupo experimental. La siguiente fase del estudio consistió en solicitar autorización a las autoridades educativas para realizar las evaluaciones de los estudiantes. Posteriormente, se solicitó a los padres de los estudiantes participantes la firma del consentimiento informado y se entregó a cada participante el asentimiento de participación a cada participante. En ese momento se procedió a las evaluaciones de los participantes. Cada evaluación fue realizada en un contexto libre de distractores y se respetaron los parámetros éticos para la investigación en los seres humanos incluidos en la declaración de Helsinki (Williams, 2008).

\section{Resultados}

En el experimento Stroop Victoria se encontró que los participantes de la muestra presentaron una media de errores por impulsividad de 83,56 $(D E 15,43)$ y una media de errores de intrusión por fallos en el control inhibitorio 2,46 ( $D E$ 2,81). En las medidas del BIS 11-c, impulsividad asociada a la planificación obtuvieron una media de 16,60 ( $D E$ 4,65), impulsividad motora 18,93 $(D E 7,42)$ e impulsividad cognitiva 7,62 (DE 2,60). En el análisis de correlación se encontró que entre los errores de impulsividad y errores por fallos en el control inhibitorio existe una relación positiva con una magnitud entre mediana $\mathrm{y}$ elevada (ver Tabla 1), lo que permite afirmar la existencia de una relación directamente proporcional entre ambas variables, ya que a mayor dificultad en la función ejecutiva control inhibitorio existiría una mayor presencia de conductas impulsivas.

Tabla 1. Coeficientes de correlación entre las medidas Stroop y BIS 11-c

\begin{tabular}{lcccc}
\hline Errores & $\begin{array}{c}\text { IMP } \\
\text { MOT }\end{array}$ & $\begin{array}{c}\text { IMP } \\
\text { PL }\end{array}$ & $\begin{array}{c}\text { IMP } \\
\text { COG }\end{array}$ & $\begin{array}{c}\text { Errores } \\
\text { por } \\
\text { intrusión }\end{array}$ \\
\hline $\begin{array}{l}\text { Errores de } \\
\text { impulsividad }\end{array}$ & $.25^{*}$ & -.02 & -.13 & $.64 * *$ \\
$\begin{array}{l}\text { Errores por } \\
\text { intrusión }\end{array}$ & .13 & -.14 & -.09 & - \\
$\begin{array}{l}\text { ** La correlación es significativa al nivel .01 } \\
\text { (bilateral) } \\
* \text { La correlación es significante al nivel .05 } \\
\text { (bilateral) }\end{array}$
\end{tabular}

Los errores de impulsividad del experimento Stroop correlacionaron en forma positiva y en una magnitud pequeña con la subescala del BIS 11-c de impulsividad motora (ver Tabla 1). En el resto de medidas del experimento y las subescalas del BIS 11-c no se obtuvieron relaciones estadísticamente significativas.

En el análisis de chi cuadrado se encontró que no existe asociación entre los errores de impulsividad $\left.\left(x^{2}[261], \mathrm{N}=81\right]=262,99, p=.45\right) \mathrm{y}$ errores por fallos del control inhibitorio $\left(x^{2}[90\right.$, $\mathrm{N}=81]=83,25, \quad p=.68) \quad$ con la edad de los participantes.

En la comparación de las dos variables del experimento Stroop Victoria, mediante el procedimiento U de Mann-Whitney, considerando al género de los participantes como factores, se encontró que no existen diferencias en errores por impulsividad ( $U=709,00 p=.39$ ), ni en errores de intrusión por fallos en el control inhibitorio $(U=695,50 \quad p=.31)$. En la Figura 4 se puede observar los puntajes por género.

\section{Discusión}

El objetivo del presente artículo fue adaptar el experimento Stroop en su versión Victoria al 


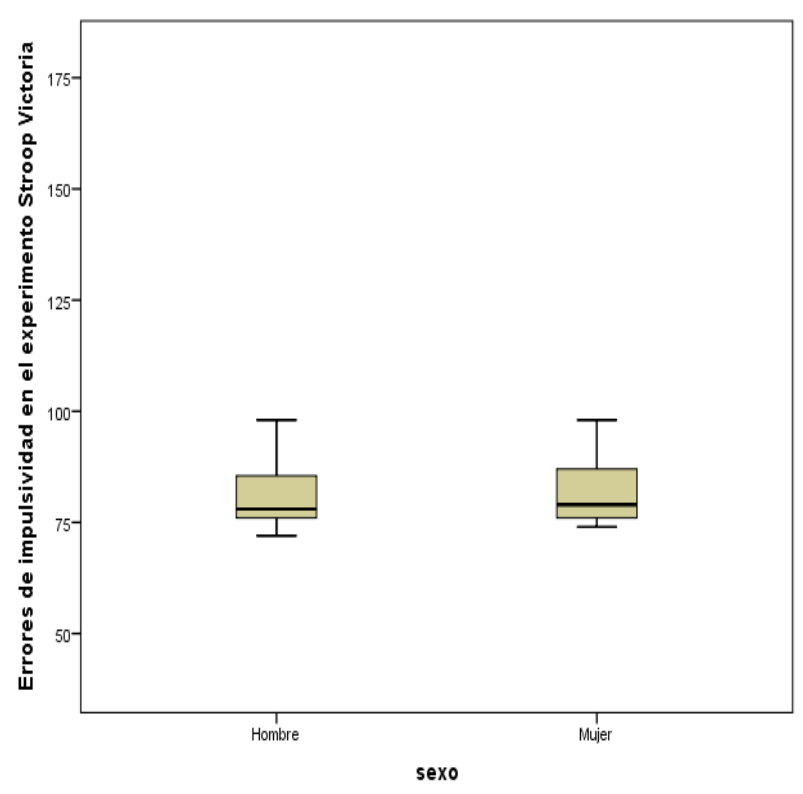

Figura 4. Puntajes obtenidos por hombres y mujeres en la variable errores de impulsividad en el experimento Stroop Victoria

contexto ecuatoriano. En función de dicho objetivo se adaptó el contenido lingüístico del experimento y se lo aplicó a un grupo de estudiantes de Ecuador, se analizó la correlación entre las medidas del experimento y la escala BIS 11-c, además, se relacionó el rendimiento de los participantes con el género y la edad.

Se pudo verificar una relación importante entre los errores de impulsividad y los errores por fallos inhibitorios, resultado evidencia convergente respecto de lo reportado por Charchat-Fichman y Martins (2009) y Musso, (2009). Sin embargo, la magnitud de asociación encontrada en el presente estudio, permite identificar que ambos constructos serían entidades independientes. Este resultado tiene sentido con el postulado teórico propuesto por dos autores clásicos en el estudio de las funciones ejecutivas, Norman y Shallice (1986), quienes afirman que el control del comportamiento puede darse en tres niveles, uno totalmente automático, otro semi consciente y otro totalmente deliberado. En los dos primeros niveles actúan un mecanismo denominado dirimidor de conflictos, mientras que en el tercer nivel actúan las funciones ejecutivas como el control inhibitorio, en un mecanismo denominado sistema atencional supervisor.

Por tanto, cuando los participantes cometieron errores por impulsividad se encontrarían actuando bajo su dirimidor de conflictos, ya que en dicho tipo de respuestas existe un comportamiento de tipo automático o semiconsciente, ya que no inhiben una tendencia de acción impulsiva. Los errores por intrusión por fallos en el control inhibitorio podrían explicarse por algún déficit en el sistema atencional supervisor, ya que los participantes responden en base al contenido literal de la palabra y no por su color, es decir, respondieron sin inhibir la tendencia automática a leer la palabra, y no seleccionando el color de la tinta con la que estaba escrita la palabra.

Además, los errores por intrusión podrían presentarse porque el individuo actúa mediante estructuras cerebrales implicadas con funciones cognitivas básicas, mientras que, cuando es capaz de inhibir la tendencia automática a la lectura, actúa mediante estructuras cerebrales frontales, implicadas en redes funcionales que permiten la praxis de habilidades mentales de alta complejidad, como el control inhibitorio (Luria, 1984; Shallice, Stuss, Picton, Alexander, \& Gillingham, 2008).

En la relación entre las subescalas del BIS 11c y las medidas del experimento Stroop Victoria se encontró, únicamente, relación entre errores de impulsividad del Stroop y la subescala de impulsividad motora. Este hallazgo tendría sentido porque ambos constructos pertenecerían al sistema denominado dirimidor de conflictos (Norman \& Shallice, 1986), puesto que estarían supeditados a un comportamiento automático o semiconsciente. Por su parte, los errores de intrusión estarían asociados al sistema atencional supervisor, relacionado con las funciones ejecutivas, las cuales no consideradas en la escala BIS 11-c.

$\mathrm{Al}$ igual que lo que ocurre con los estudios de Malek et al (2013) y Charchat-Fichman y Martins (2009), en el cual evaluaron una muestra de edad similar a la del presente estudio, no se han encontrado que las variables del experimento (errores por impulsividad y errores por fallos en el control inhibitorio) puedan estar asociadas al género de los participantes o que existan diferencias entre los mismos.

Sin embargo, de forma contradictoria, en el estudio de Moering, Schinka, Mortimer y Graves (2004), se encontraron que las respuestas en el experimento Stroop si estarían relacionadas con el género y la edad de los participantes, no obstante, 
en dicho estudio se investigó a una muestra de sujetos adultos mayores, a diferencia del presente estudio que fue realizado con estudiantes de 6 a 15 años de edad.

Una de las principales limitaciones del estudio realizado consiste en el tamaño de muestra y su procedencia (una determinada ciudad de Ecuador). Esto no permite la generalización de los resultados, sin embargo, se convierte en un factor de motivación para la realización de una siguiente investigación donde se pueda considerar una muestra representativa de Ecuador.

Como investigación futura es de interés analizar la capacidad discriminativa del experimento Stroop Victoraia, con la finalidad de diferenciar entre estudiantes con desarrollo típico y estudiantes con trastornos del neurodesarrollo y del aprendizaje, como el trastorno por déficit de atención con hiperactividad, agresividad, bajo desempeño académico, entre otros.

\section{Agradecimientos}

El investigador del presente estudio expresa su gratitud a: (1) la Secretaria de Educación Superior, Ciencia, Tecnología e Innovación (SENESCYT) del Ecuador por el apoyo económico recibido dentro de la beca para cursar estudios de $\mathrm{PhD}$ en Psicología que financió el presente proyecto y (2) al Profesor PhD Shane Muller por su gentil respuesta ante las dudas que se presentaron en la programación del experimento.

\section{Referencias}

Anderson, P., \& Reidy, N. (2012). Assessing executive function in preschoolers. Neuropsychol Rev, 22, 345-360.

Baddeley, A. (2012). Working memory: Theories, models, and controversies. Annual Review of Psychology, 63, 1-29.

Barkley, R. (2011). Avances en el diagnóstico y la subclasificación del trastorno por déficit de atención/hiperactividad: Qué puede pasar en el futuro respecto al DSM-V. Revista de Neurología, 48 (2), 101-106.
Brown, T. (2008). ADD/ADHD and impaired executive function in clinical practice. Current Psychiatry Reports, 10(5), 407-411.

Chahin, N., Cosi, S., Lorenzo-Seva, U., \& VigilColet, A. (2010). Stablity of the factor structure of Barrat's Impulsivity Scales for children across cultures: A comparison of Spain and Colombia. Psicothema, 22(4), 983989.

Charchat-Fichman, H., \& Martins, R. (2009). Perfromance of 119 brazilian children on Stroop Paradigm-Victoria Version. Arquivos de Neuro-Psiquiatria, 6(2-B), 445-449.

Damasio, A. (1994). El error de Descartes. Santiago de Chile: Editorial Andrés Bello.

García-Gómez, A. (2015). Desarrollo y validación de un cuestionario de observación para la evaluación de las funciones ejecutivas en la infancia. Revista Intercontinental de Psicología y Educación 17(1), 141-162.

Gioia, G., Isquith, P., Guy, S., \& Kenworthy, L. (2000). Behavior Rating Inventory of Executive Function. Lutz, Florida: Psychological Assessment Resources.

Gioia, G., Isquith, P., Retzlaff, P., \& Espy, K. (2002). Confirmatory factor analysis of the Behavior Rating Inventory of Executive Function (BRIEF) in a clinical sample. Child Neuropsychology, 8(4), 249-257.

Goldberg, E. (2002). El cerebro ejecutivo. Lóbulos frontales y mente civilizada. Barcelona: Editorial Crítica Drakontos.

Hernández, R., Fernández, C., \& Baptista, P. (2014). Metodología de la Investigación. Sexta edición. México DF: Mc Graw Hill Education.

Lezak, M. (1995). Neuropsychological Assessment, 3th Edition. Oxford: University Press.

Luria, A. (1984). El cerebro en acción. Barcelona: Editorial Martínez Roca.

Malek, A., Hekmati, I., Amiri, S., Pirzadeh, J., \& Gholizadeh, H. (2013). The Standarization of Victoria Stroop Color-Word Test among iranian bilingual adolescents. Archives of Iranian Medicine, 16(7), 380-384.

Moering, R., Schinka, J., Mortimer, J., \& Graves, A. (2004). Normative data or elderly africanamericans for the Stroop Color and Word 
Tests. Archives of Clinicla Neuropsychology, 19, 477-586.

Mueller, S. (2015). The psychology experiment building language (Version 0.14) [Software]. Disponible en $\mathrm{m}$ http://pebl.sourceforge.net.

Mueller, S., \& Piper, B. (2014). The Psychology Experiment Building Language (PEBL) and PEBL Test Battery. Journal of Nueroscience Methods, 250 - 259.

Musso, M. (2009). Evalaución de funciones ejecutivas en niños: Análisis y adaptación de pruebas en un ocntexto escolar. Revista Iberoamericana de Diagnóstico y Evaluación - e Avaliação Psicológica, 27(1), 157-178.

Norman, D., \& Shallice, T. (1986). Attention to action: Willed and automatic control of behavior. En R. Davidson, G. Schwartz, \& D. Shapiro, Consciousness and self-regulation (pp. 1-18). New York: Editorial Plenumm Press.

Ramírez, V., \& Rosas, R. (2007). Test de inteligencia para niños de Wechsler. Santiago de Chile: Ediciones Universidad Católica.

Ramos, C., Guevara, M., Bolaños, M., \& Ramos, D. (2015). Adaptación y estudio descriptivo del experimento Go/No-Go en una muestra de estudiantes ecuatorianos. Revista Tecnológica, 28(2), 119-133.

Rubiales, J. (2014). Perfil ejecutivo en niños con trastorno por déficit de atención con hiperactividad. Revista Iberoamericana de Diagnóstico y Evaluación - e Avaliação Psicológica, 38(2), 31-54.

Shallice, T., Stuss, D., Picton, T., Alexander, M., \& Gillingham, S. (2008). Mapping task switching in frontal cortex through neuropsychological group studies. Frontiers in Neuroscience, 2, 79-85.

Stanford, M., Mathias, C., Dougherty, D., Lake, S., Anderson, N., \& Patton, J. (2009). Fifty years of the Barratt Impulsiveness Scale: An update and review. Personality and Individual Differences, 47, 385-395.

Troyer, A., Leach, L., \& Strauss, S. (2006). Aging and response inhibition: Normative data for the Victoria Stroop Test. Aging, Neuropsychology, and Cognition, 13, 20-35.

Verbruggen, F., Logan, G., \& Stevens, M. (2008). STOP-IT: Windows executable software for the stop-signal paradigm. Behavior Research Methods, 40(2), 479-483. 